LA-7809-MS

Informal Report
Effect of the Gas Blanket on the Stability of the Dense Z-Pinch 
LA-7809-MS

Informal Report

Special Distribution

Issued: May 1979

\section{Effect of the Gas Blanket on the Stability of the Dense Z-Pinch}

B. R. Suydam

This report was prepered as an eccuunt of work

sponsored by the United States Goveminent. Neither the

United States nor the Unted States Departmant of

Enerar

Energ, nor any of their employees. nor any of their

ches

any warranty. express or implied, or assumes any legal

liability or responsibility for the accuracy, completeness

or use fulness of any information, apparatus, produet of

process disclosed, $r$, represents that its use would not

infringe privately owned ights.

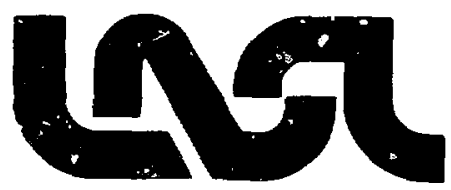




\title{
EFFECT OF THE GAS BLANKET ON THE STABILITY \\ OF THE DENSE Z-PINCH
}

\author{
by
}

B. R. Suydam

\begin{abstract}
We here stuciy the stabilizing effect of the dense neutral gas blanket which surrounds the dense zpinch as it is presently operated. Because this gas can contribute to pressure balance, plasma pressure can fall to zero at some radius without introducing destabilizing surface currents which would be required for pressure balance without the gas. Thus pressure profiles are possible which are stable against interchange, and long wavelength kink modes are more easily stabilized by a wall. Internal kink modes are unaffected and are unstable.
\end{abstract}

Introduction

The dense z-pinch [1] differs sharpiy from conventional pinches in two ways. First, the medium surrounding the p? asma is not vacuum but is rather a dense neutral gas. In the second place, the time scale of the experiment, combined with the interaction between the plasma and its gas blanket. most probably ensures that there is no period of the phenomenon which could be described as quasi static.

In this report we are going to concentrate our attention on the first of the above differences and ignore the other. The question we shall ask is the following: Given a static cylindrical z-pinch plasma suriounded by a dense neutrai gas, what is the effect of the gas blanket on the stability of this system? We shall consider the plasma to te an ideai magneto fluid. Stability will be studied by applying the energy principle of Bernstein et al. [2] A trivial modicication of one of their energy integrals includes the neutral gas blanket into the thecry. In Section 1 we write out the energy 
integrals and, after a small manipulation, throw the theory into a form in which the precise nature of the stabiiizing effect of the neutral bianket is clearly manifest.

In Section 2 we study the mode known variously as the $m=0$ mode, the sausage mode, or the interchange mode. Kadont.sev [3] has given a criterion for stability of this mode which we re-derive in order to correct a typographical error in Ref. [3]. We find that, generally speaking, the interchange mode can be stabilized provided the pressure of the neuiral gas blanket is high enough.

In Section 3 we study the kink modes, i.e. the modes for which $m \neq 0$, and find that a conducting wall outside the gas blanket stabilizes long wavelength modes but not the short wavelengths.

1. The Energy Principie

As our model, we consider a volume $V_{p}$, bounded by the surface $\sum$, filled with an ideal perfectly conducting magnetized fluid, the plasma. Surrounding the plasma is a region $v_{g}$ of neutral gas and of magnetic field. Clearly $v_{g}$ is bounded on its inside by the surface $\sum$; it may also be bounded on the outside by a wall, $\Sigma_{W}$, or it may extend to infinity. To study the stability of this system we first construct the energy integral.

As the neutral gas and the magnetic field are totally uncoupled in $V_{g}$ we can at first ignore the gas. Then we can write down immediately the energy integral for the system plasma plus surrounding magnetic field as given by Bernstein et al. [2] We have

$$
\text { (1.1) } \delta W_{1}=\delta W_{p}+\delta W_{\Sigma 1}+\delta W_{B},
$$

where

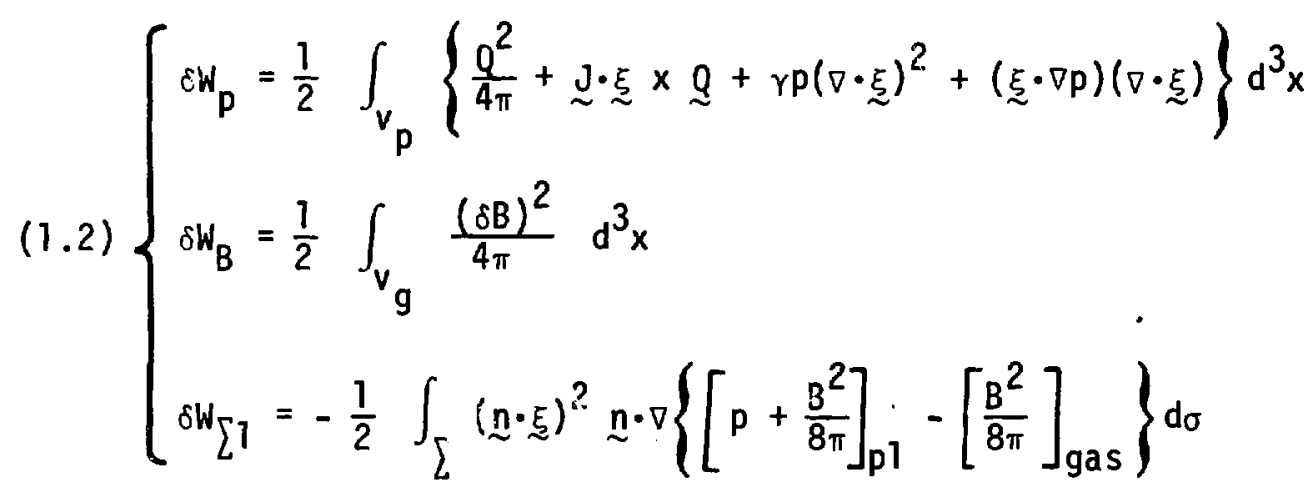

where $\mathrm{n}$ is the unit normal to $\sum$ pointing away from the plasma. 
The three expressions of Eq. (1.2) are respectively the contribution of the magnetized plasma, the contribution of the surrounding $B$-field, and the contribution of the plasma-vacuum interface. In the vacuum (or gas) $\underset{\sim}{\delta B}$ is the perturbed $B-f i e l d$ and it is, of course, a vacuum field. In the plasma wo have set

(1.3) $\underset{\sim}{Q} \equiv \underset{\sim}{\delta B}=\operatorname{curl}(\underset{\sim}{\xi \times B})$.

Now let us add the gas. Clearly, it gives a second contribution containing both a volume and a surface term

$$
(1.4) \quad \delta W_{2}=\delta W_{V}+\delta W_{L 2}
$$

For $\delta W_{V}$ and $\delta W_{\Gamma 2}$ we can simply copy Eqs. (1.2) remembering now that $\delta B$ shouid be set zero as it has already been taken care of, and $\underset{\sim}{n}$ is replaced by $\underset{\sim}{\sim}$, as $\underset{\sim}{n}$ points into the gas. Thus

$$
\text { (1.5) } \delta W_{V}=\frac{1}{2} \int_{V_{g}}\left[\gamma p(\nabla \cdot \underset{\sim}{\xi})^{2}+(\underset{\sim}{\xi} \nabla p)(\nabla \cdot \underset{\sim}{\xi})\right] d^{3} x
$$

and

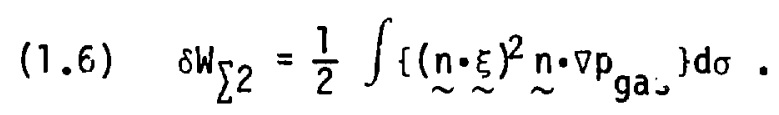

For the total energy integral we have merely to add together the above results and obtain

$$
\text { (1.7) } \delta W_{T}=\delta W_{p}+\delta W_{g}+\delta W_{\Sigma},
$$

where

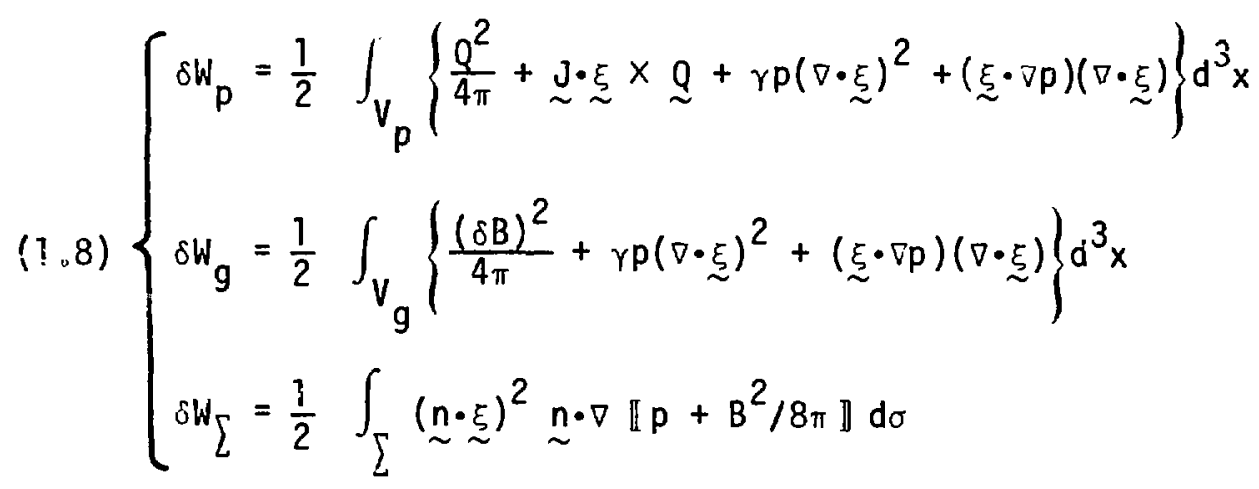

where for any quantity $X, \llbracket X \rrbracket$ means the jump in $x$, namely,

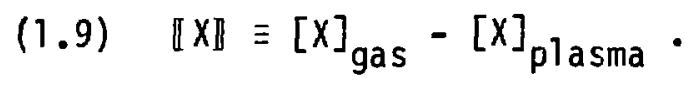


If we remember that in the gas $\underset{\sim}{J}=0$ whereas, in the $\mathrm{plasma} \underset{\sim}{\delta B}=\underset{\sim}{Q}=\operatorname{curl}(\underset{\sim}{\xi \times B})$, we see that the expressions for $\delta W_{p}$ and $\delta W_{g}$ are formally identical. The difference is in the coupling between $\underset{\sim}{\xi}$ and $\underset{\sim}{\delta}$ in the $p l a s m a$. Hote that we write no contribution for the wali as this is supposed to be rigid, i.e. $\underset{\sim}{n} \cdot \underset{\sim}{\xi}$ vanishes on the wall, or else to be at infinity where everything vanishes.

We are by definition studying a static equilibrium; therefore, in the gas the unperturbed pressure $p$ must be uniform. Therefore, $\underset{\sim}{\xi} \cdot \nabla p \equiv 0$ and $\delta W_{g}$ is minimized with respect to $\underset{\sim}{\xi}$ by choosing

$$
(1.10) \quad 7 \cdot \xi=0 \text { in } V_{g} \text {. }
$$

With this choice the second of Eqs. (1.8) reduces to

$$
(1.11) \delta W_{g}=\frac{1}{2} \int_{V_{g}} \frac{(\delta B)^{2}}{4 \pi} d \sigma .
$$

Thus so far, the gas makes no contribution, stabilizing nor destabilizing. Equation (1.10) in no way limits our ability to choose $\underset{\sim}{n} \underset{\sim}{\underline{\xi}}$ arbitrarily on $\sum$.

Next we look at the surface term. As we envisage a plasma pressure which does not vanish on $\sum$, $\sum$ must be a flux surface, al though for our purpose there need be no others. It is convenient to calculate in orthogonal curvilinear coordinates $\left(x^{1}, x^{2}, x^{3}\right)$ so chosen that the surface $\sum$ has the equation $x^{1}=a$ constant and that $x^{1}$ increases going from plasma to gas. Then we have

$$
\text { (1.12) } \underset{\sim}{\mathrm{n}} \underset{\sim}{\mathrm{B}} \equiv \mathrm{B}_{1}=0 \text { on } \sum \text {. }
$$

The metric we write in the usual way, namely,

$$
(1.13)(d s)^{2}=\left(h_{1} d x^{1}\right)^{2}+\left(h_{2} d x^{2}\right)^{2}+\left(h_{3} d x^{3}\right)^{2} \text {. }
$$

In these coordinates we can write the 1-component of the pressure balance equations as

$$
\text { (1.14) } \begin{aligned}
\frac{1}{h_{1}} \partial_{1}\left\{p+\frac{B^{2}}{8 \pi}\right\} & =\frac{1}{4 \pi}\left\{(B \cdot \nabla) B_{1}+B_{1}\left[B_{2} \frac{\partial_{2} h_{1}}{h_{2} h_{1}}+B_{3} \frac{\partial_{3} h_{1}}{h_{3} h_{1}}\right]\right. \\
& \left.-B_{2}^{2}\left(\frac{{ }_{1} h_{2}}{h_{1} h_{2}}\right)-B_{3}^{2}\left(\frac{{ }_{1} h_{3}}{h_{1} h_{3}}\right)\right\},
\end{aligned}
$$


where the $h^{\prime}$ s are all defined by Eq. (i.13). Now on $\sum, B_{1}$ vanishes and so, therefore, does $\underset{\sim}{(B \cdot \nabla) B_{7}}$. Therefore, on $[\mathrm{Eq} .(1.14)$ reduces to

$$
\text { (1.15) } \stackrel{n}{\sim} \cdot \nabla\left[p+\frac{B^{2}}{8 \pi}\right]_{p i}=-\frac{1}{4 \pi}\left\{B_{2}^{2}\left(\frac{{ }^{2} h_{2}}{k_{1} h_{2}}\right)+B_{3}^{2}\left(\frac{\partial_{1} h_{3}}{h_{1} h_{3}}\right)\right\} \text { (on } \sum \text { ). }
$$

In the gas $p$ is a constant and

$$
\text { (1.16) } \stackrel{n}{\sim} \cdot \nabla\left[p+\frac{B^{2}}{8 \pi}\right]_{\text {gas }}=\frac{1}{4 \pi h_{1}}\left[B_{1} \partial_{1} B_{1}+B_{2} \partial_{2} B_{2}+B_{3} \partial_{3} B_{3}\right] \text {. }
$$

But in the gas we have a vacuum field, hence

$$
\text { (1.17) }\left\{\begin{array}{l}
\partial_{1}\left(h_{2} B_{2}\right)=\partial_{2}\left(h_{1} B_{1}\right)=0 \text { on } \sum \\
\partial_{1}\left(h_{3} B_{3}\right)=\partial_{3}\left(h_{1} B_{1}\right)=0 \text { on } \sum
\end{array}\right.
$$

Expanding out the 1 aft-hand sides of Eqs. (1.17) and substituting into Eq. $(1.16)$ evaluated on $\sum$ we find

$$
\text { (1.18) } \underset{\sim}{n \cdot \nabla} \sim\left[\hat{p}+\hat{B}^{2} / 8 \pi\right]_{\text {gas }}=-\frac{1}{4 \tau}\left\{\hat{B}_{2}^{2} \frac{\partial_{1} h_{2}}{h_{1} h_{2}}+\hat{B}_{3}^{2} \frac{\partial_{1} h_{3}}{h_{1} h_{3}}\right\} \quad \text { (on }[\text { ), }
$$

where we have put hats on quantities pertaining to the gas. Our surface energy termi now becomes

$$
\text { (1.19) } \left.\delta W_{\sum}=\frac{1}{8 \pi} \int_{\sum} \underset{\sim}{(n-\xi)}\right)^{2}\left\{\left(B_{2}^{2}-\hat{B}_{2}^{2}\right) \frac{\partial_{1} h_{2}}{h_{1} h_{2}}+\left(B_{3}^{2}-\hat{B}_{3}^{2}\right) \frac{{ }_{1} h_{3}}{h_{1} h_{3}}\right\} h_{2} h_{3} d x^{2} d x^{3} .
$$

On the surface $\sum,\left(x^{2}, x^{3}\right)$ form an orthogonal coordinate system with metric $\left(h_{2}, h_{3}\right)$. The normal curvature of the $x^{2} 1$ iries is just $-\partial_{1} h_{2} /\left(h_{1} h_{2}\right)$ and that of the $x^{3}$ lines is $-\partial_{1} h_{3}\left(h_{1} h_{3}\right)$. Thus the quantities $-\partial_{1} h_{2} /\left(h_{1} h_{2}\right)$ and

$-\partial_{1} h_{3}\left(h_{1} h_{3}\right)$ are the principal curvatures of the surface; their product is the Gaussian curvature and their sum is the mean curvature. From Eq. (1.19)

follow the usual thecrems about effects of magnetic line curvature on stability. This is discussed in the Appendix.

We see from Eq. (1.19) just what the effects of a neutral gas blanket can be. In the absence of such a blanket $\hat{p}=0$ and if $p \neq 0$ on $\sum$, 
then $\hat{B}^{2}>B^{2}$ ecessarily. Thus if $\sum$ is convex, $\delta W_{\sum}$ is negative for some modes. However, when $\hat{p}=p$ on $\sum$ then no surface current is required by pressure balance and $\underset{B}{B}=\underset{\hat{B}}{\hat{B}}$ on $\sum$. It then follows that

If there are no surface currents, $p$ ind $\underset{\sim}{B}$ are continuous across $\sum$ and

(1.20) $\delta W_{\Sigma}=0$.

It is precisaly in this surface term and nowhere else that the gas blanket has its stabilizing effect.

2. The interchange $(m=0)$ Modes

In cylindrical geumetry nothing in the equilibrium state depends on $\theta$ nor on $z$ so we can Fourier analyze, setting

(2.1) $\underset{\sim}{\xi}=\underset{\sim}{\xi i}(r) \exp [i k z+i m \theta]$.

As $B_{r}=B_{z}=0$ for the $z$-pinch, $Q$ simplifies to

(2.2) $\left\{\begin{array}{l}Q_{r}=(i m / r) \xi_{r} B, \\ Q_{\theta}=-\sigma_{r}\left(\xi_{r} B\right)-i k \xi_{z} B, \\ Q_{z}=(i m / r) \xi_{z} B,\end{array}\right.$

where $B$ means $B_{\theta}$. In the gas $\underset{\sim}{\delta}$ is a vacuum field and therefore satisfies

$$
\text { (2.3) }\left\{\begin{array}{l}
(m / r) \delta B_{z}-k \delta B_{\theta}=0, \\
i k \delta B_{r}-\partial_{r}\left(\delta B_{z}\right)=C, \\
(1 / r) \partial_{r}\left(r \delta B_{r}\right)+i(m / r) \delta B_{\theta}+i k \delta B_{z}=0 .
\end{array}\right.
$$

There is a third curl $\underset{\sim}{\delta B}$ equation but it is satisfied by virtue of the first two. We can use che first two of Eqs. (2.3) to el iminate $\delta B_{\theta}$ and $\delta B_{r}$ from the third, which then becomes

$$
\text { (2.4) } \frac{d^{2}\left(\delta B_{z}\right)}{d r^{2}}+\frac{1}{r} \frac{d\left(\delta B_{z}\right)}{d r}-\left(k^{2}+\frac{m^{2}}{r^{2}}\right) \delta B_{z}=0,
$$

which is just Bessel's equation. Froin this we can calculate

$$
\text { (c.5) } \delta B_{r}=-(i / k) a_{r}\left(\delta B_{z}\right) \text {. }
$$


The boundary condition is that the perturbed vacuum field be tangent to the perturbed boundary, which simply reduces to

$$
\text { (2.6) } Q_{r}=\delta B_{r} \text { at } r=R
$$

in our case.

For $m=0, \delta B_{\theta}$ vanishes and so does $Q_{r}$; thus $\delta B_{r}$ vanishes on

$r=R$. Therefore, $\underset{\sim}{\delta B}=0$ satisfies Eqs. (2.3) and the boundary condition (2.6) and moreover minimizes $\delta W_{g}$, so we have

$$
\text { (2.7) } \delta H_{g}=0 \text {. }
$$

As we saw in the last section, in the absence of a surface current sheet we also have

$$
\text { (2.8) } \delta W_{\Sigma}=0 \text {, }
$$

so the only contribution to $\delta \mathrm{W}$ is that of the $\mathrm{plasma}$ and we can now apply the analysis of Kadomtsev [3]. As this analys is depends in no way on any boundary condition we see that as far as the stability of the $m=0$ modes is concerned, the neutral gas blanket is equivalent to a rigid wall. This equivalence vanishes when $m \neq 0$. Kadomtsev's analysis has indeed been applied [1] to assert the existence of interchange stable configurations; our Eqs. (2.7) and (2.8) prove this application to be sound.

In Ref. [3] there is an unfortunate typographical error in the final result. As the analys is is not long, we therefore reproduce it here, correcting the error. For $m=0$ Eqs. (2.2) can be written

$$
\text { (2.9) } Q_{r}=0=Q_{2} ; Q_{\theta}=-B(\nabla \cdot \underset{\sim}{\xi})-\xi_{r}\left(B^{\prime}-B / r\right) \text {, }
$$

where the prime means $d / d r$. From this a short calculation yields

$$
\text { (2.10) } \begin{aligned}
\delta W=\delta W_{p} & -\frac{1}{2} \int_{0}^{R}\left\{\left[\gamma p+B^{2} / 4 \pi\right]|\nabla \cdot \xi|^{2}-\frac{2 B^{2}}{4 \pi r}[\underset{\sim}{\xi} \underset{\sim}{\xi} \underset{\sim}{\xi}+c c]\right. \\
& \left.+\frac{2 B}{4 \pi r}\left[\frac{B}{r}-B^{\prime}\right]\left|\xi_{r}\right|^{2}\right\} 2 \pi r d^{2},
\end{aligned}
$$

where the star * means complex conjugate and "cc" stands for the complex conjugate of the preceeding term. Now 


$$
\text { (2.11) } \nabla \cdot \xi_{\sim}=(1 / r) \partial_{r}\left(r \xi_{r}\right)+i k \xi_{z}
$$

and, therefore, we can choose $\xi_{r}$ and $\nabla \cdot \xi$ as our inciependent variables rather than $\xi_{r}$ and $\xi_{z}$. When we do so we immediately see that the choice

$$
\text { (2.12) } \nabla \cdot \underset{\sim}{\xi}=\frac{2 B^{2} /(4 \pi r)}{\gamma p+B^{2} / 4 \pi} \xi_{r}
$$

minimizes $\delta W$ with respect to $\nabla \cdot \underset{g}{\xi}$. This minimum value is

$$
\text { (2.13) } \delta W=\int_{0}^{R} \frac{B^{2}}{2 r}\left\{\frac{\gamma p-B^{2} / 4 \pi}{\gamma p+B^{2} / 4 \pi}-\frac{r B^{\prime}}{B}\right\}\left|\xi_{r}\right|^{2} d r \text {. }
$$

As $\xi_{r}$ is completely arbitrary, it follows that the system is stable against interchange provided

$$
\text { (2.14) } \frac{\gamma p-B^{2} / 4 \pi}{\gamma p+B^{2} / 4 \pi} \geqslant \frac{r B^{\prime}}{B}
$$

at every point within the plasma, and, otherwise, it is unstable. Kadomtsev writes

$$
\text { (2.15) } B=8 \pi p / B^{2}
$$

and uses the pressure balance equation to eliminate $B^{\prime}$. Then condition (2.14) takes the form

(2.16) $\frac{-r p^{\prime}}{p} \leqslant \frac{4 \gamma}{\gamma \beta+2}$.

It is clear from Eq. (2.14) that the condition

$$
\text { (2.17) } r B^{\prime} / \tilde{B} \leqslant 1 \text {, }
$$

with equality allowed only at $r=0$, is necessary for stability. In a sense it is also sufficient. More precisely stated we have the following result.

If the current density $J_{2}$ decreases monotonically as $r$ increases, then a positive constant $p_{1}$ exists such that replacing $p$ by $p+p_{1}$ everywhere renders the system stable against interchange. Kadomtsev does not state this result, but it clearly follows from Eq. (2.14). Equation (2.14) clearly shows that the higher the pressure in the gas blanket the better the chance for interchange stability. 
Kadomtsev [3] interprets the stability condition by examining the marginal case in which inequality is replaced by equality in Eq. (2.16). He notes also that pressure balance can be written in the form

$$
\text { (2.18) } \frac{r p^{\prime}}{p}=\frac{r \beta^{\prime}-2 \beta}{\beta(1+\beta)} \text {. }
$$

Combining this with (2.16) written as an equality yields

$$
\text { (2.19) } r \beta^{\prime}=-\frac{2 \beta[\gamma \beta+2(\gamma-1)]}{\gamma \beta+2} \text {. }
$$

Next note that

$$
\text { (2.20) } r p^{\prime}=r \frac{d p}{d \beta} \frac{d \beta}{d r}=-\frac{2 \beta[\gamma \beta+2(\gamma-1)]}{\gamma \beta+2} \frac{d p}{d \beta}
$$

and set this into condition (2.16) written as an equality. The result is

$$
\text { (2.21) } \beta[\gamma \beta+2(\gamma-1)] \frac{d p}{p d \beta}=2 \gamma \text {, }
$$

which may be integrated to yịeld

$$
\text { (2.22) } p=p_{0}\{\beta /[\beta+2(\gamma-1) / \gamma]\}^{\frac{\gamma}{\gamma-1}},
$$

where $p_{0}$ is the integration constant; it is the pressure on axis. This, with general $\gamma$, is the first of Kadomtsev's Eqs. (4.4) (p. 166 of Ref. [3]). We can also integrate Eq. (2.19) and obtain

$$
\text { (2.23) } r=a\left\{\frac{[\beta+2(\gamma-1) / \gamma]^{2-\gamma}}{\beta}\right\} \frac{1}{2(\gamma-1)} \text {. }
$$

Again we have an integration constant, a. This is how the second of Kodomtsev's Eqs. (4.4) should read, after setting $\gamma=5 / 3$. Equation (2.23) gives the profile of $\beta(r)$ and Eqs. (2.22) and (2.23), together, give the profile $p(r)$ in parametric form. From these we can easily construct profiles of the magnetic field and of the current density. At large $r$ Eqs. (2.22), (2.23), and (2.15) give the following asymptotic expressions:

$$
\text { (2.24) }\left\{\begin{array}{l}
p \sim p_{0}[\gamma / 2(\gamma-1)]^{\gamma}(a / r)^{2 \gamma}, \\
B \sim[\gamma / 2(\gamma-1)] \sqrt{3 \pi p_{0}}(a / r), \\
B \sim[2(\gamma-1) / \gamma]^{2-\gamma}(a / r)^{2(\gamma-1)}
\end{array}\right.
$$


The criticai pressure profîe fails off rather slowiy with increasing $r$. Anything falling ofi more rapidly is unstable.

3. The Kink Modes, $m \neq 0$

When $m \neq 0$ Eqs. (2.2) must be used as written, but we are permitted the simplification of restricting our attention to modes for which $\nabla \cdot \xi \underset{\xi}{\xi}=0$. Then one can minimize $\delta W_{p}$ algebraically with respect to $\xi_{\theta}$ and $\xi_{z}$ and reduce the plasma integral to

$$
\text { (3.1) } \delta W_{p}=\frac{1}{4} \int_{0}^{R}\left\{\frac{\left(r f \xi_{r}^{\prime}+g \xi_{r}\right)^{2}}{m^{2}+k^{2} r^{2}}+\left(f^{2}-h\right) \xi_{r}^{2}\right\} r d r \text {, }
$$

where for a general cylindrical pinch

$$
\text { (3.2) } \begin{cases}f=k B_{z}+m B_{\theta} / r & g=k B_{z}-\mathrm{mB}_{\theta} / r \\ h=\left(8 \pi J_{z} / r\right) B_{\theta} & \xi_{r}^{\prime}=d \xi_{r} / d r\end{cases}
$$

In our case $B_{z}=0$ so $f=-g=m B_{\theta} / r$ and $E q$. (3.1) becomes

$$
\delta W_{p}=\frac{1}{4} \int_{0}^{R}\left\{\frac{m^{2} B^{2}\left(\xi_{r}^{\prime}-\xi_{r} / r\right)^{2}}{m^{2}+k^{2} r^{2}}+\left[\frac{m^{2}-2}{r^{2}} B^{2}-\frac{2 B B^{\prime}}{r}\right] \xi_{r}^{2}\right\} r d r .
$$

In examining only modes for which $\nabla \cdot \underset{g}{\xi}$ vanishes we explicitly exclude the $m=0$ modes. Therefore, $\delta W_{p}$ is clearly minimized with respect to $m^{2}$ for $m^{2}=1$; the worst $k$ modes are clearly $k^{2}=\infty$. For these worst modes we have

$$
\text { (3.4) }\left.\delta W_{p}\right|_{k^{2}=\infty} ^{m=r}=-\frac{1}{4} \int_{0}^{R}\left[2 B B^{\prime}+B^{2} / r\right]\left(\xi_{r}\right)^{2} d r,
$$

whence it follows that if

$$
\text { (3.5) } 2 \mathrm{BB}^{\prime}+\mathrm{B}^{2} / \mathrm{r}>0 \text {, }
$$

anywhere within the $p l a s m a ~ \delta W_{p}$ can be made negative for all suitably large values of $k^{2}$. Moreover, it can be made negative by a $\xi$ which vanishes at $r=R$ and hence does not perturb anything outside the plasma. These large $k$ modes cannot be stabilized by any outside influences. Now $B$ is zero at $r=0$, increases to a maximum and ultimateiy will fall off as $r^{-1}$. Throughout the 
region from $r=0$ to that point where $B$ falls as $r^{-1 / 2}$ Eq. (3.5) is satisfied, so this whole inner region is kink unstable to short wavelength perturbations. Note that this unstable region extends well beyond where $B_{\theta}$ reaches its maximum value, so we cannot expect a very large stabilization of these modes by finite Larmor orbit effects. The growth of kinks will produce a $\mathrm{B}_{\mathrm{z}}$, which may have a stabilizing effect. Note that we can rewrite Eq. (3.5) as a condition for stability (reversed inequality) in the form

$$
(3.5)^{\prime} \frac{r p^{\prime}}{p}-\frac{r \beta^{\prime}}{\beta}+1<0 \text {. }
$$

If we now examine Kadomtsev's marginally interchange stable profiled we can substitute into Eq. (3.5)', Eq. (2.16) written with equality and Eq. (2.19). For this profile we find that the kink stability condition becomes

$$
\text { (3.6) } \beta \leqslant 2 /(3 \gamma) \text {. }
$$

Thus a hardcore z-pinch is possible, which is completely MHD stable.

It riay be instructive to examire also the limit of very long wavelengths, i.e. the $k=0$ modes. Writing out Eq. (3.3) for $m=1, k=0$

$$
\text { (3.7) } \begin{aligned}
\delta W_{p} & =\frac{1}{4} \int_{0}^{R}\left\{B^{2} r\left(\xi_{r}^{\prime}\right)^{2}-2 B^{2} \xi_{r} \xi_{r}^{\prime}-2 B B^{\prime} \xi_{r}^{2}\right\} d r \\
& =\frac{1}{4} \int_{0}^{R} r B^{2}\left(\xi_{r}^{\prime}\right)^{2} d r-\frac{1}{4}\left[B^{2} \xi_{r}^{2}\right]_{r=R} .
\end{aligned}
$$

If $\xi_{r}(R)=0, \underset{\sim}{\delta B}=0$ and $\delta W=\delta W_{p}$ and is clearly positive. Dtherwise, $\delta W_{p}$ is clearly minimized by the choice $\xi_{r}=$ const. and gives

$$
\text { (3.8) } \delta W_{p}=-\frac{1}{4}\left[B^{2} \xi_{r}^{2}\right]_{r=R} \text {. }
$$

As always in our examples $\delta W_{Y}=0$, but there will be a contribution from the gas. The first two of Eqs. (2.3) simply give $\delta B_{z}=0$ when $k=0$, so we need the third curl equation, namely

$$
\text { (3.9) } \frac{1}{r} \partial_{r}\left(r \delta B_{\theta}\right)-\frac{i}{r} \delta B_{r}=0 \text {. }
$$

The third of Eqs. (2.3) can be solved for $\delta B_{\theta}$ and the result set into Eq. (3.9) to give

$$
\text { (3.10) } r \frac{d^{2}}{d r^{2}}\left(\delta B_{r}\right)+3 \frac{d}{d r}\left(\delta B_{r}\right)=0 .
$$


This can readily be sclived to give

$$
\delta B_{r}=A\left[1-\left(R_{0} / r\right)^{2}\right]
$$

$$
\delta B_{\theta}=i A\left[1+\left(R_{0} / R\right)^{2}\right] .
$$

One of our integration constants is $A_{;}$the other has been chosen so that $\delta B_{r}$ vanishes on a rigid wall at $r=R_{0}$. If now we use the boundary condition of Eq. (2.6) and use the first of Eqs. (2.2) we find

$$
\text { (3.12) } A=-\frac{i R B}{R_{0}^{2}-R^{2}} \xi_{r} \text {. }
$$

From Eq. (3.11)

$$
(\delta B)^{2}=2 A^{2}\left[1+\left(\frac{R_{0}}{r}\right)^{4}\right],
$$

whence

$$
\text { (3.13) } \begin{aligned}
\delta \omega_{g} & =\int_{R}^{R} \frac{(\delta B)^{2}}{8 \pi} 2 \pi r d r=\frac{1}{4} A^{2}\left(R_{0}^{2}-R^{2}\right)\left(R_{0}^{2}+R^{2}\right) / R^{2} \\
& =\frac{1}{4} B^{2} \xi_{r}^{2}\left(\frac{R_{0}^{2}+R^{2}}{R_{0}^{2}-R^{2}}\right) .
\end{aligned}
$$

Adding together

$$
\text { (3.14) } \delta W=\delta W_{p}+\delta W_{g}=\frac{1}{4} B^{2} 5_{r}^{2}\left(\frac{2 R^{2}}{R_{0}^{2}-R^{2}}\right) .
$$

Thus the system is always stable for $k=0$, approaching neutral stability as the wall recedes to infinity $\left(R_{0} \rightarrow \infty\right)$.

In review, then, kink modes behave as follows. When $k=0$ the mode is stable even for a wall at infinity. As $k$ increases, the wall must be brought nearer and nearer to the plasma in order to stabilize it. At some critical value $k_{c}$ neutral stability is first achieved by a wall at the plasma surface, and for all $k>k_{c}$ internal kink modes are unstable. 
Conclusions

We have shown that a neutral gas blanket has a stabilizing effect on a magnetically confined plasma. If the plasma is surrounded by vacuum, and if its pressure drops abruptly to zero at the plasma-vacuum interface, then a surface current is necessary to maintain pressure equilibrium. If at any point of the plasma surface any confining B-line curves towards the plasma, this surface current is destabilizing. The effect of a neutra? gas blanket is precisely this, that its pressure can balance the plasma pressure and thus eliminate the destabilizing surface current.

As applied to the dense z-pinch, the neutral gas blanket makes possible plasma distributions which are stable against the interchange, or sausage, modes. What is required is that the plasma pressure fall of not too rapidly in radius. A rigid conducting wall bounding the gas will stabilize long wavelength but not short wavelength kink modes. At very short wavelength the worst modes are internal kink modes. As this unstable interior extends well beyond the radius of maximum $B_{\theta}$, it is unlikely that finite Larmor orbit effects will help much.

As normally formed, the dense z-pinch generally heats up more rapidly than the confining field builds up, with the result that the plasma column expands and sends a shock wave out into the surrounding gas bianket. This can in no way affect the internal kink modes, but the back pressure of the shock would, according to our analysis, tend toward stabilizing the interchange modes.

References

1. Jay E. Hanmel "An Ohmically Heated High-Density Z-Pinch" Los Alamos Scientific Laboratory Report LA-6203-MS, January 1976.

2. I. B. Bernstein, E. A. Frieman, M. D. Kruskal, and R. M. Kulsrud "Än Energy Principle for Hydromagnetic Stability Problems", Proc. Roy. Soc. A 244, pp. $17-40,1958$.

3. B. B. Kadomtsev, "Hydromagnetic Stability of a Plasma", in Reviews of Plasmã P'lysics, Editor M. A. Leontovich, Vol. 2, pp. 165-168, Consultants Bureau, New York, 1966. 


\section{APPENDIX}

\section{SOME GEOMETRY}

As in Section (1), we let $\left(x^{1}, x^{2}, x^{3}\right)$ represent orthogonal curvilinear coordinates such that, our flux surface $\sum$ has the equation $x^{1}=$ const and that $x^{1}$ increases going from plasma to gas. Let $\tau_{(i)}$, be the unit tangent to the $x^{i}$ coordinate line. Then the principal curvature of this line, ${ }_{\sim}^{k}(i)$ is given by

$$
\text { (A.1) } \underset{\sim}{\kappa}(i)=\underset{\sim}{(\tau(i)} \stackrel{\sim}{\sim} \underset{\sim}{\tau}(i)=[\operatorname{curl} \underset{\sim}{\tau}(i)] \times \underset{\sim}{\tau}(i)
$$

or in components,

$$
(A .2)\left\{\begin{array}{l}
\underset{\sim}{\sim}(1)=\left(0,-\frac{\partial_{2} h_{1}}{h_{2} h_{1}},-\frac{\partial_{3} h_{1}}{h_{3} h_{1}}\right) \\
\underset{\sim}{\sim}(2)=\left(-\frac{\partial_{1} h_{2}}{h_{1} h_{2}}, 0,-\frac{\partial_{3} h_{2}}{h_{3} h_{2}}\right) \\
\underset{\sim}{\sim}(3)=\left(-\frac{\partial_{1} h_{3}}{h_{1} h_{3}}, \frac{\partial_{2} h_{3}}{h_{2} h_{3}}, 0\right)
\end{array}\right.
$$

and these hold, of course, throughout the space in general and over $\sum$ in particular. As the unit normal to $\sum$ has components $(1,0,0)$ we see that on $\sum$ we have

$$
\text { (A.3) } \left.\left.\quad-B_{2}^{2} \frac{\partial_{1} h_{2}}{h_{1} h_{2}}-B_{3}^{2} \frac{\partial_{1} h_{3}}{h_{1} h_{3}}=B_{2}^{2} \underset{\sim}{(\underset{n}{n} \cdot \underset{\sim}{k}(2)}\right)+B_{3}^{2} \underset{\sim}{(\underset{n}{n} \cdot \underset{\sim}{k}(j)}\right) .
$$

Clearly on $\sum \underset{\sim}{n \cdot \underset{3}{n}}(2)$ and $\underset{\sim}{n \cdot k} \underset{\sim}{(3)}$ are the normal curvatures, respectively, of the $x^{2}$ and of the $x^{3}$ coordinate lines. As our cocrdinate system is orthogonal, these coordinate lines are also lines of curvature so these two normal curvatures are indeed the principal curvatures. 
Now let us write $\underset{\sim}{\mathrm{B}}=\underset{\sim}{\mathrm{Bb}}$, where $B$ is the magnitude and $\underset{\sim}{\mathrm{b}}$ is a unit vector. Then we compute

$$
\text { (A.4) } \quad \underset{\sim}{(B} \cdot \nabla) \underset{\sim}{B}=B^{2}(\underset{\sim}{b} \cdot \nabla) \underset{\sim}{b}+\underset{\sim}{B b}(\underset{\sim}{b} \cdot \nabla) B \text {. }
$$

Now clearly, the principal curvature of the $B-l$ ines, $\underset{\sim}{K}(b)$, is given by

$$
\text { (A.5) } \underset{\sim}{\kappa}(b)=\underset{\sim}{(b} \cdot \nabla) \underset{\sim}{b},
$$

whence, evaluating Eq. (A.4) on $\sum$ and scalar multiplying by $\underset{\sim}{\mathrm{n}}$ we get

$$
\text { (A.6) } \quad \underset{\sim}{n} \cdot(\underset{\sim}{B} \cdot \nabla) \underset{\sim}{B}=B^{2}(\underset{\sim}{n} \cdot \underset{\sim}{K}(b)) .
$$

But we could also expand $\underset{\sim}{B}$ on $\sum$ as

$$
\text { (A.7) } \underset{\sim}{\mathrm{B}}=\mathrm{B}_{2 \sim} \tau(2)+\mathrm{B}_{3} \tau(3)
$$

Using this we get, on $\sum$,

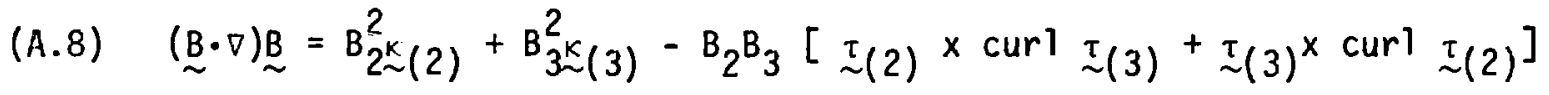

$$
\begin{aligned}
& +\underset{\sim}{\tau}(2)\left[B_{2}(\tau(2) \cdot \nabla) B_{2}+B_{3}(\stackrel{\tau}{\sim}(3) \cdot \nabla) B_{2}\right] \\
& +\tau(3)\left[B_{2}(\tau(2) \cdot \nabla) B_{3}+B_{3}(\tau(3) \cdot \nabla) B_{3}\right] .
\end{aligned}
$$

Scalar multiplying this by $\underset{\sim}{n}$ we obtain

$$
\text { (A.9) } \quad \underset{\sim}{n} \cdot(\underset{\sim}{B} \cdot \nabla) \underset{\sim}{B}=B_{2}^{2}(\underset{\sim}{n} \cdot \underset{\sim}{*}(2))+B_{3}^{2}(\underset{\sim}{n} \cdot \underset{\sim}{\sim}(3))
$$

as one easily sees that $\underset{\sim}{\sim} \cdot \tau(2) \times$ curl $\underset{\sim}{\sim}(3)=0=\underset{\sim}{n} \cdot \tau(3) \times$ curl $\underset{\sim}{\sim}(2)$.

Combining Eqs. (A.9) and(A.6) with (A.3) we get

$$
\text { (A.10) } \left.\quad-B_{2}^{2} \frac{\partial_{1} h_{2}}{h_{1} h_{2}}-B_{3}^{2} \frac{\partial_{1} h_{3}}{h_{1} h_{3}}=B^{2} \underset{\sim}{(\underset{\sim}{n} \cdot \underset{\sim}{*}(b)}\right) \text {, }
$$

where $\underset{\sim}{n} \cdot \mathfrak{\sim}_{b}$ is, of course, the normal curvature of the $B-l$ ines which make up the surface $\sum$. 
In the general case where surface currents are allowed $\underset{\sim}{B}$ is not continuous across the surface $\sum$ and the plasma $\underset{\sim}{B}-1$ ines are not the same as the gas $\underset{\sim}{\hat{B}}-1$ ines. Let us denote by $\kappa_{n}$ the normal curvature of the $\underset{\sim}{B}-l$ ines (the plasma field) on $\sum$ and by $\hat{\kappa}_{n}$ the normal curvature of the gas $\hat{B}-7$ ines. Then Eq. (1.19) can be written equivalently as

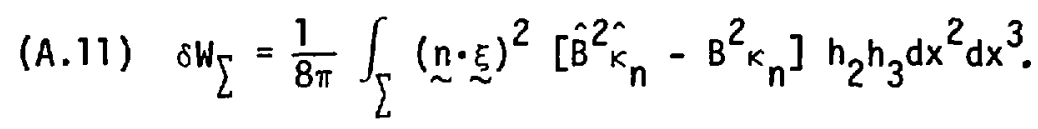

As a special case consider a completely diamagnetic plasma inside of which $B=0$. Then Eq. (A.11) says that such a plasma is unstable if there is any portion of $\sum$ over which $\hat{\kappa}_{n}$ is negative, i.e. for which the center of principal curvature of the $\hat{B}-l$ ines lies on the plasma side of $\sum$. For we merely choose $(\underset{\sim}{n} \cdot \underset{\sim}{\xi}) \neq 0$ over such a region and zero el sewhere and $\delta W_{\Sigma}$ is negative. The above is not yet quite a proof. We must add to it the remarks:

a.) Because $p=$ const, $B=0$ in the plasma, the choice $(\nabla \cdot \xi)=0$ in the plasma reduces $\delta W_{p}$ to its minimum value, zero.

b.) The vacuum magnetic field always gives a non-negative contribution but as shown in [1] $\underset{\sim}{n} \cdot \xi$ on $\sum$ can always be chosen so that $\left|\delta W_{g}\right|<<\left|\delta W_{\Sigma}\right|$ so the sign of $\delta W_{\Sigma}$ really tells the whole story. 